\title{
Research on the Denoise Method of Speckle Image Contour and Background
}

\author{
Junyao, Yin, Xinglong Zhu* \\ College of Mechanical Engineering, Yangzhou University, Yangzhou, China \\ *Corresponding Author : xlzhu@yzu.edu.cn
}

\begin{abstract}
When the laser spot is irradiating directly on the surface of the measured object, the accuracy of the center coordinate value (CCV) of the speckle will affect the depth information. A lens imaging model of monocular vision and laser spot combination was established, the analytical relationship between $\mathrm{CCV}$ of the speckle and depth information was derived, the causes of speckle contour noise and background noise were expounded, the denoise method was proposed and the corresponding denoise algorithm was designed. Finally, the simulation verifies that the proposed method is feasible by theoretical data, and the proposed algorithm enables to ensure the robustness of $\mathrm{CCV}$ of the speckle.
\end{abstract}

Keywords: speckle contour, algorithm, ellipse fitting, denoise.

\section{Introduction}

Ellipse fitting has a wide range of applications in many areas, such as pattern recognition and computer vision $^{(1)}$, biological cell segmentation ${ }^{(2)}$ and so on. As an application, there are many algorithms for ellipse fitting. However, they are usually divided into three categories: least square method, Hough transform method and edge contour tracking method. The least squares method can be divided into two categories according to the optimized function, which are respectively the method based on the minimum geometric error and the method based on the smallest algebraic error ${ }^{(3)}$. The method based on the minimum geometric error can accurately fit the ellipse ${ }^{(4)}$, while there are also some shortcomings in this kind of method. It needs multiple iterations in solving the nonlinear optimization problem and is very sensitive to noise. This method cannot guarantee convergence, and convergence is related to the initial iteration value. The method based on the smallest algebraic error is easy to implement and its calculation efficiency is high. However, the main problem is that this kind of method cannot guarantee that the fitting result is ellipse and some additional conditions are needed. For example, the penalty function method proposed by Szpak $^{(5)}$ adopts Sampson distance to guarantee ellipse fitting. Despite this, the result still has deviation. This method is less robust than the method based on the minimum geometric error, especially when the fitting data comes from a small part of the ellipse. The accuracy of this method also depends on the selection of the initial iteration value. The Hough transform is also one of the most well-known methods for detecting ellipse ${ }^{(6)}$ and there are many improved methods based on it ${ }^{(7)}$. These methods are susceptible to noise in the image. The advantage of the Hough transform method is that the coherent edge pixels do not need to be continuous, which is very useful when the observable data is a sparse set of points. However, its disadvantage is that it is too sensitive for parameter space quantization selection and incorrect quantization may result in false ellipse or loss of true ellipse. When the number of ellipses in the image increases, the performance of the Hough method deteriorates. The edge tracking method studies continuous edge pixels to detect ellipses ${ }^{(2,8-9)}$. The main strategy of this type of method is to detect arcs and then group them. Chia ${ }^{(8)}$ improved the above algorithm by introducing autocorrelation and the grouping phase was corrected by feedback. The low-confidence ellipse would be replaced by a series of imaginary ellipses, which were composed of different sets of arcs. This method is very successful for multi-ellipse detection in digital images. However, the method is only useful for continuous edge pixels. If the observable sample data is a sparse point set, 
this method is incomplete. Dilip ${ }^{(9)}$, Ratko ${ }^{(10)}$ separately used edge curvature and convexity, used the multidimensional model of GMM increasing contour pixel position and orientation information, used classical least squares method combined with RANSAC method to solve the problem of multi-elliptic identification. Peng ${ }^{(11)}$ proposed a method for measuring the internal defects of objects based on laser speckle tangential interferometry. Mahsa ${ }^{(12)}$ improved curve fitting by using speckle, which heightened the accuracy of absolute displacement measurement.

The above methods have achieved good results on different occasions. However, the laser speckle is scattered, its background is noisy and the surface has defects in the actual situation. The speckle image obtained by CCD will have small contour, edge noise and background noise after image processing. The center position of the speckle image will be offset, which will affect the measurement accuracy. In view of the influence of the small contour, edge noise and background noise, this paper proposes a method to eliminate the small contour, edge noise and background noise. Theoretical data is used to verify the feasibility of the proposed method and algorithm, so as to ensure the robustness of CCV of the speckle.

\section{Lens imaging model}

\subsection{Description of lens imaging model}

The monocular vision is combined with the laser spot to measure and the laser spot is used as an active marker point to illuminate the measured surface. As shown in Figure1, the position information of marker point changes on the CCD speckle image, which can help us obtain the distance information.

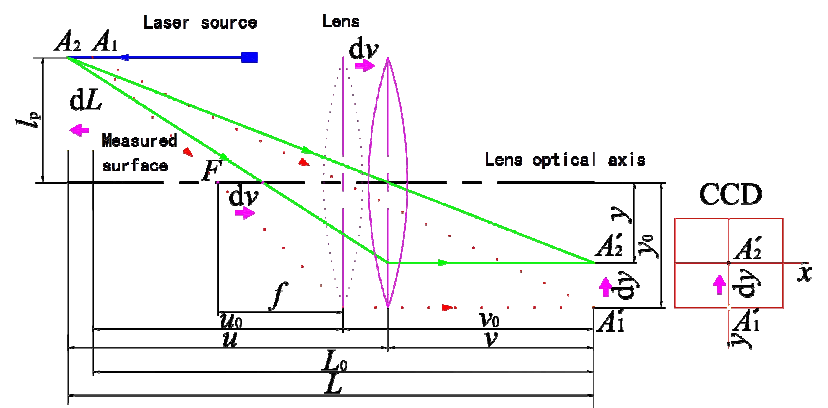

Fig. 1. Lens imaging model

$L_{0}$ is the distance between the point $A_{1}$ on the measured surface and the CCD surface. $L$ is the distance between the point $A_{2}$ and the CCD surface after the movement of the measured surface. $\mathrm{d} L$ is the distance difference before and after the movement of the measured surface. $l_{p}$ is the distance between the axis of the light source and the optical axis of the lens. $y_{0}$ is the imaging position of point $A_{1}^{\prime}$ on the imaging surface. $y$ is the imaging position of point $A_{2}^{\prime}$ on the imaging surface. $\mathrm{d} v$ is the distance of the lens movement. $u_{0}, v_{0}, u, v$ respectively represent the object distance and subject distance before and after lens adjustment. $f$ is the focal length of lens.

In Fig. 1, the CCD surface of the monocular vision camera and the laser source are symmetrically biased arranged on both sides of the lens axis, which makes the axis of the laser source, the lens axis and the $\mathrm{Y}$ axis of the CCD surface are located in the same plane. Therefore, when the measured object moves, the CCV of the speckle is changed on the $\mathrm{Y}$ axis of the CCD surface and unchanged on the $\mathrm{X}$ axis of the CCD surface. Under this circumstance, the model can be simplified. The lens is connected to the mover of the DC servo motor to adjust the lens position. When the position of the measured surface changes (recorded as $\mathrm{d} L$ ), the object distance becomes larger and the image distance does not change. The lens imaging rule is destroyed and the speckle image becomes blurred. At this time, the lens position is adjusted by the DC servo motor (the moving distance is recorded as $\mathrm{d} v$ ), the speckle image is changed to clear and the $\mathrm{CCV}$ of the new speckle image $y$ is recorded after image processing.

It can be seen from the analysis in Figure 1 that if the initial position obeys the lens image rule, then we have

$$
L_{0}=\frac{f l_{p}}{y_{0}}+\frac{f}{l_{p}} y_{0}+2 f
$$

According to formula (1), when the initial location $L_{0}$ is known, $y_{0}$ can be obtained, and $y_{0}$ is referred to as the datum point.

Let $L_{0}=4 f$, and get $y_{0}=l_{p}$ from formula (1).

When the object moves, the imaging speckle becomes blurred. The lens position is adjusted to make the imaging speckle clear, and the lens image formation rule is obeyed.

$$
L=\frac{f l_{p}}{y}+\frac{f}{l_{p}} y+2 f
$$

Subtracting the formula (1) by the formula (2), the depth information difference is:

$$
\mathrm{d} L=L-L_{0}=f\left(\frac{l_{p}}{y_{0} y}-\frac{1}{l_{p}}\right)\left(y_{0}-y\right)
$$

According to formula (3), when the installation of the measurement system is completed and the initial position is determined, the change of depth information $\mathrm{d} L$ is only related to the CCV of the imaging speckle $y$. If the CCV of the imaging speckle $y$ has disturbance, the measurement 
accuracy of the depth information will be directly affected.

Considering $y_{0}=l_{p}$, formula (3) can be simplified into formula (4).

$$
\mathrm{d} L=f\left(\frac{1}{y}-\frac{1}{l_{p}}\right)\left(l_{p}-y\right)=f \frac{\left(y_{0}-y\right)^{2}}{y_{0} y}
$$

It can be seen from the above analysis that how to accurately obtain the coordinate of the imaging speckle center and whether the coordinate of the imaging speckle center can truly reflect the location of the measured points determine the accuracy of the depth information acquisition.

\subsection{The cause of noise in imaging speckle images}

\section{1) Background noise}

Ideally, there is no luminous point can match the laser brightness in the field view of the camera, and the measurement surface is absolutely flat. Under this circumstance, the speckle image acquired by the camera goes through the image processing and only the speckle image contour left. The result is shown in Fig. 2. However, there always be environmental factors such as light, daylight and reflective objects within the field view of the camera in actual situations, and the brightness is equivalent to the brightness of the laser spot. So it can be seen from Fig. 3 that the contours of these interference points can still remain after the image processing. Even if the imaging speckle image goes through the image processing by using the region of interest (ROI), there will still appear the contours of the interference points in the ROI. The result is shown in Fig. 4. The above interference point contour is called the background noise.

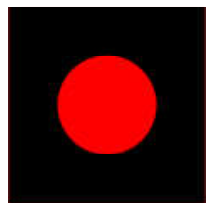

(a) Speckle image

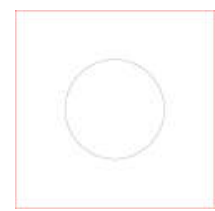

(b) Contour image
Fig. 2. Ideal background

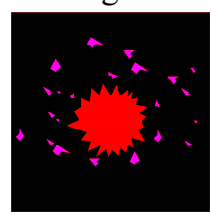

(a) Speckle image

(b) Contour image

Fig. 3. Actual background

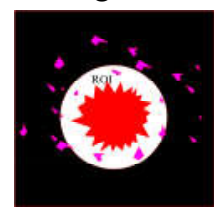

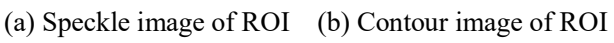

Fig. 4. Actual background of ROI

\section{2) Contour noise}

When the laser irradiates the surface of the object, scattering occurs due to surface roughness. It can be seen from Fig. 5 that some scattered irregular light spots are generated around the contour of the imaging speckle.

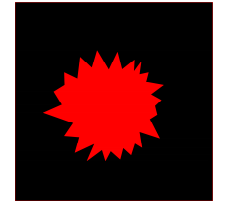

(a) Speckle image (b) Speckle contour image

Fig. 5. Laser speckle image

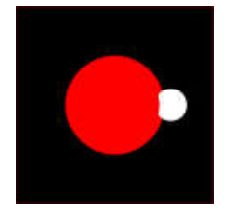

(a) Pit image

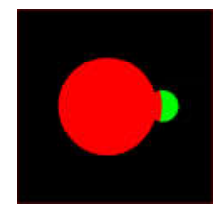

(c) Bulge image

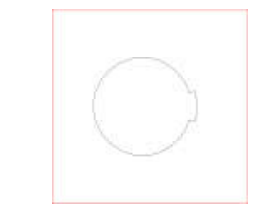

(d) Bulge contour

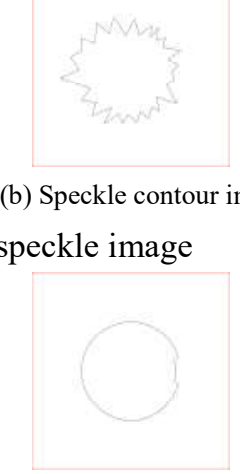

(b) Pit contour

Fig. 6. Laser speckle images of pits and bulges

When there are defects such as pits or bulges on the surface of the object and the speckle is irradiated on the pits or bulges, some noise is generated at the edge of the speckle formed on the CCD surface. The results are shown in Fig. 6.

\section{Research on noise elimination methods}

From the above analysis, it is known that both background noise and speckle contour noise will affect the $\mathrm{CCV}$ of the speckle. Therefore, it is necessary to deal with the background noise and the edge noise of the speckle image. Then, the least squares ellipse fitting method is used to obtain the CCV of the speckle.

\subsection{Imaging background noise elimination method}

As shown in Fig. 7 a), the speckle image of the measured surface is collected. Fig. 7 b) shows the result of extracting the edge contour of the speckle image. There may be a number of small edge contours caused by the background in the extracted edge contour. For Fig. 7 b), the area of the extracted multiple edge contour is calculated. If the area of contour is less than a certain threshold, the contour should be eliminated (the threshold is determined by a reference to the area of the speckle image). It can be seen from Fig. 7 c) that only the speckle contour of ROI and the large background contour noise are left after the above processing. 


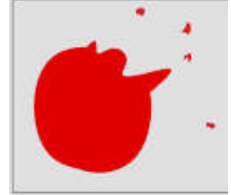

(a) Speckle image

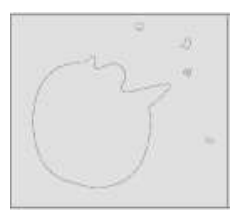

(b) Contour image

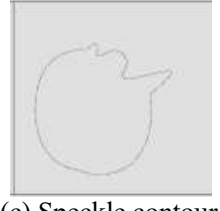

(c) Speckle contour
Fig. 7. Speckle image processing

\subsection{Contour noise elimination method}

As shown in Fig. 7 c), due to the influence of pits, bulges and surface roughness, there is noise in the extracted speckle edge contour. The edge noise also affects the CCV of the speckle. Therefore, the edge noise needs to be processed.

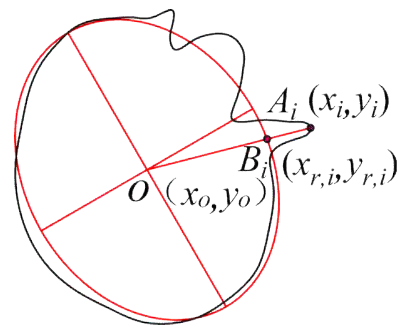

Fig. 8. Denoise method of speckle contour

Fig. 8 illustrates the elimination method of edge noise from speckle contour. Using the least squares ellipse fitting method, the elliptic equation and the coordinate values of the elliptical center point $O\left(x_{o}, y_{o}\right)$ are obtained. The contour point $A_{i}\left(x_{i}, y_{i}\right)$ and the elliptical center point $O\left(x_{o}, y_{o}\right)$ are selected to establish the equation of a straight line $O A_{i}$. Jointly with the fitting elliptic equation, the intersection point $B\left(x_{\mathrm{r}, i}, y_{\mathrm{r}, i}\right)$ with the fitting ellipse is obtained, and then what should be solved is:

$$
\begin{gathered}
\left|O B_{i}\right|=\sqrt{\left(x_{o}-x_{\mathrm{r}, i}\right)^{2}+\left(y_{o}-y_{\mathrm{r}, i}\right)^{2}}, \\
\left|O A_{i}\right|=\sqrt{\left(x_{o}-x_{\mathrm{i}}\right)^{2}+\left(y_{o}-y_{\mathrm{i}}\right)^{2}} 。
\end{gathered}
$$

Firstly, we define $\lambda_{i}=\left|O A_{i}\right| /\left|O B_{i}\right|$. If $\lambda_{i}=1$, it means that the contour point falls on the fitting ellipse. If $\lambda_{i}>1$, it means that the contour point falls outside the fitting ellipse. If $\lambda_{i}<1$, it means that the contour point falls inside the fitting ellipse. Then, $\left|\lambda_{i}-1\right|$ is defined as the degree that the contour discrete point deviates from the fitting ellipse. In order to avoid eliminating the possible effective points, $\left|\lambda_{i}-1\right|$ is sorted in the algorithm design. A certain number of contour points with the largest absolute value of $\left|\lambda_{i}-1\right|$ are defined as contour noise, and the noise should be eliminated. Then, the edge points that have gone through the elimination of edge noise are used again for fitting until the CCV of the fitting ellipse converges.

\subsection{Algorithm design}

According to the elimination methods mentioned above, the algorithm flow chart is shown in Fig. 9. The equation of the straight line $O A_{i}$ can be described as: $\frac{x-x_{o}}{x_{o}-x_{i}}=\frac{y-y_{o}}{y_{0}-y_{i}}$, The fitting elliptic equation can be described as: $x^{2}+k_{1} x y+k_{2} y^{2}+k_{3} x+k_{4} y+k_{5}=0$ 。

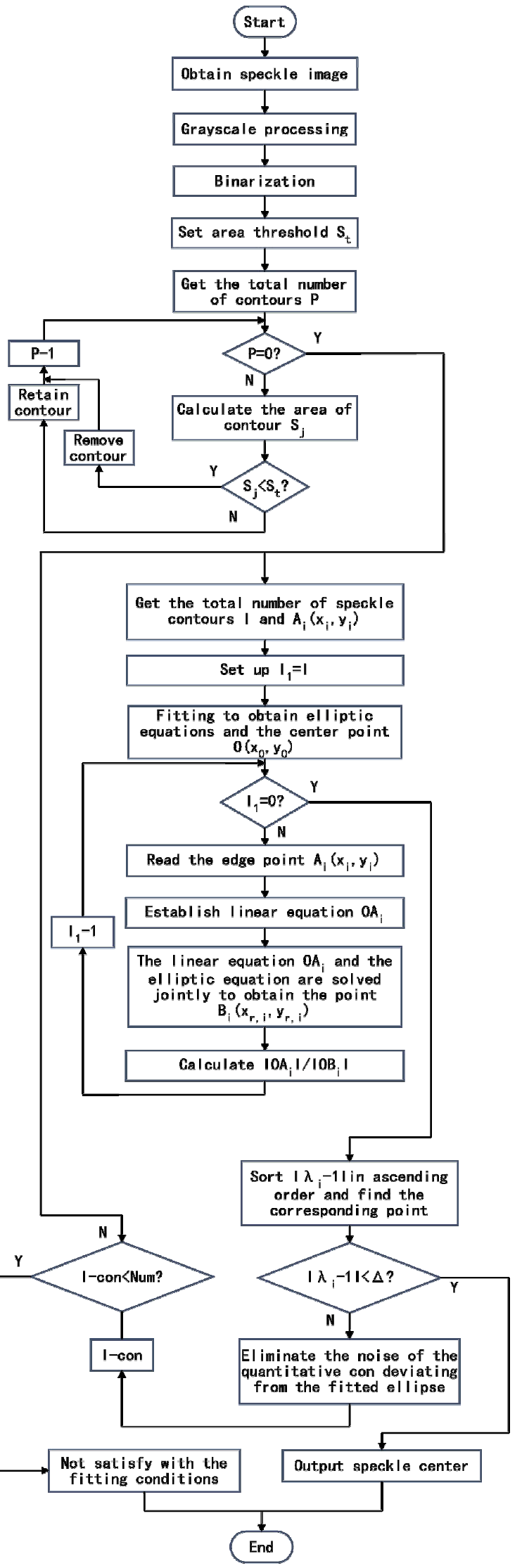

Fig. 9. Algorithm flow chart 
$p$ is the number of contour and $S_{t}$ is the threshold set by referring the area of laser speckle. $S_{j}$ is the area of small contour and $I$ is the total number of speckle contour points. $I_{1}$ is the intermediate variable and used for temporary storage of $I . \Delta$ is the control value of the edge noise deviating from the fitting ellipse. If all $\left|\lambda_{i}-1\right|$ are less than $\Delta$, the algorithm ends and the CCV of the fitting ellipse is output as the center position of the speckle image. con is the number of eliminated edge noise after each round of fitting and the total number of the next round of fitting ellipse points is reduced to $I-c o n$. Num is set as the minimum value of the total number of edge points. If the total number of edge points is less than Num, then the loop iteration will end.

Through the image processing of the image speckle, the edge contour of the speckle is obtained. Then, the contour data is fitted through the least square ellipse, and the center point of the fitting ellipse is considered as the center point of the speckle.

\section{Theoretical data simulation}

As shown in Fig. 10 a), the ideal speckle image with noise consists of ellipse (red), pit (cyan), bump (green), and background light source (pink). The center of the ellipse is at $(10,10) \mathrm{mm}$. Fig. $10 \mathrm{~b}$ ) shows that the speckle contour is obtained by image processing. It is shown in Fig. $10 \mathrm{c}$ ) that the edge contour points should be extracted according to a certain rule because of many contour discrete points. The ellipse fitting algorithm is applied to the discrete points in Fig. $10 \mathrm{c}$ ) and points should be eliminated quantitatively in every round (this paper eliminates 2 points per round). The convergence process of eliminating the edge noise and the background noise is shown in Fig. 10 d) to Fig. 10 m).

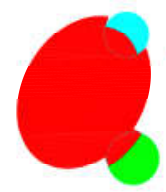

a) Speckle

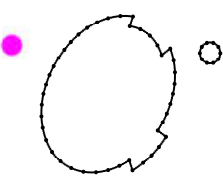

b) Contour c) Discrete point

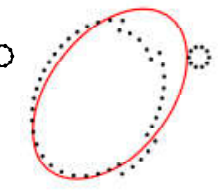

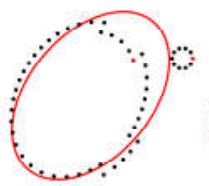

d)

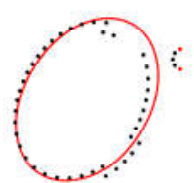

h)

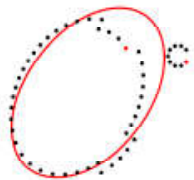

e)

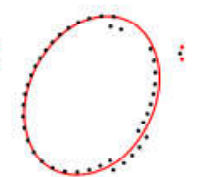

i)

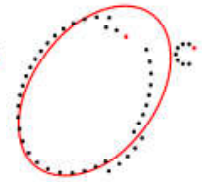

f)

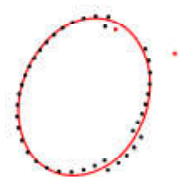

j)

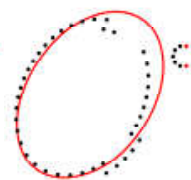

g)

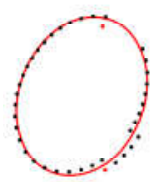

k)

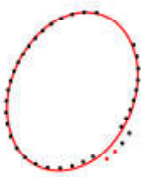

1)

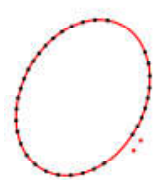

m)
Fig. 10. Denoise process of edge and background noise

As shown in Fig. $10 \mathrm{~m}$ ), during the elimination process of edge noise and background noise of the theoretical speckle, the speckle center converges to the theoretical center $(10,10) \mathrm{mm}$ after 11 iterations. The CCV of the speckle changed with the iteration rounds is shown in Table 1.

Table 1. $\mathrm{x}$ and $\mathrm{y}$ values of speckle center coordinates

\begin{tabular}{|l|l|l|l|l|l|}
\hline Round & 1 & 2 & 3 & 4 & 5 \\
\hline$x / \mathrm{mm}$ & 10.5879 & 10.5602 & 10.5349 & 10.5018 & 10.39 \\
\hline$y / \mathrm{mm}$ & 10.1952 & 10.187 & 10.1996 & 10.2008 & 10.1167 \\
\hline Round & 6 & 7 & 8 & 9 & 10 \\
\hline$x / \mathrm{mm}$ & 10.2738 & 10.13 & 10.031 & 10.0306 & 10.0132 \\
\hline$y / \mathrm{mm}$ & 10.0285 & 9.9227 & 9.8639 & 9.8954 & 9.9291 \\
\hline
\end{tabular}

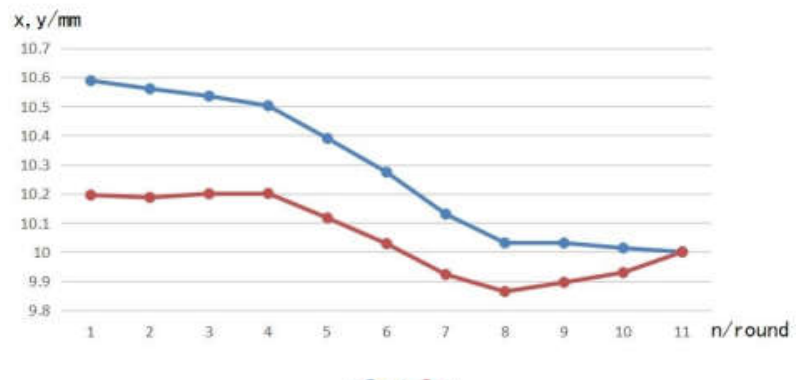

Fig. 11. Convergence diagram of theoretical fixed point eliminating noise

According to the data of Table 1, the convergence trend of the CCV of the speckle is shown in Fig. 11. It can be seen from Table 1 and Figure 11 that, as the number of iterations increases, the contour noise is gradually eliminated and the center of the speckle gradually converges to the theoretical center. It can be indicated that the algorithm is feasible in theory and lays a foundation for the next experiment verification.

\section{Conclusions}

In the monocular vision measurement system of using lens imaging model, the center position of laser speckle imaging has an important influence on measurement accuracy. In this paper, a search algorithm that quantificationally eliminates the background noise and contour edge noise is proposed. Through theoretical data 
and experimental data verification, it can effectively handle the noise, so that the CCV of the speckle tends to be stable and convergent after finite rounds. By quantitatively eliminating of the noise after finite rounds, the experimental results show that the mean or median $\mathrm{CCV}$ of the speckle can be used as the theoretical value of the speckle center, and the relative error is smaller, which can improve the measurement accuracy.

\section{Acknowledgment}

The author(s) disclosed receipt of the following financial support for the research: This work was supported by Jiangsu Province Basic Research Program Natural Science Foundation (Grant No. BK20171287 ), National Natural Science Foundation of China(Grant No. 51475409 ).

\section{References}

(1) Liu ZY, and Qiao H : "Multiple ellipses detection in noisy environments: A hierarchical approach", Pattern Recognition, Vol. 42, No. 11, pp. 2421-2433, 2009

(2) Bai X, Sun C, and Zhou F : "Splitting touching cells based on concave points and ellipse fitting", Pattern Recognition, Vol.42, No.11, pp. 2434-2446, 2009

(3) Chaudhuri D : "A simple least squares method for fitting of ellipses and circles depends on border points of a two-tone image and their 3-D extensions", Pattern Recognition Letters, Vol.31, No.9, pp. 818-829, 2010

(4) Chojnacki W, Brooks MJ and Hengel AVD : "On the Fitting Surfaces to Data with Covariances", Pattern Analysis \& Machine Intelligence IEEE Transactions on., Vol.22, No.11, 1294-1303, 2000

(5) Szpak ZL, Chojnacki $\mathrm{W}$ and Hengel AVD : "Guaranteed Ellipse Fitting with the Sampson Distance", European Conference on Computer Vision", Vol.7576, pp. 87-100, 2012

(6) Duda RO. and Hart PE : "Use of the Hough transformation to detect lines and curves in picture", Commun. ACM, Vol.15 , No.1, 11-15, 1972

(7) Nair PS and Jr ATS : "Hough transform based ellipse detection algorithm", Pattern Recognition Letters, Vol.17, No.7, 777-784, 1996

(8) Chia AY, Rahardja S, Rajan D and Leung K : "A split and merge based ellipse detector with self-correcting capability", IEEE Transactions on Image Processing A Publication of the IEEE Signal Processing Society,
Vol.20, No.7, 1991-2006, 2011

(9) Prasad DK, Leung MKH, Cho SY : "Edge curvature and convexity based ellipse detection method", Pattern Recognition, Vol.45, No.9, 3204-3221, 2012

(10) Grahovac D and Scitovski R : "A method for solving the multiple ellipses detection problem", Pattern Recognition, Vol.60, 824-834,2016

(11)Peng Y, Liu G, Quan Y and Qilin Zeng : “The depth measurement of internal defect based on laser speckle shearing interference", Optics \& Laser Technology, Vol.92, 69-73,2017

(12)Farsad M, Goch G and Evans C : "Application of correlation curve fitting to improve the absolute displacement measurement using speckle correlation, Precision Engineering-Journal of the International Societies for Precision Engineering And Nanotechnology, Vol.46, 129-134, 2016 